\title{
Screening Echocardiogram in High-Risk Women with Class III Obesity to Predict the Risk of Preeclampsia
}

\author{
Maeve K. Hopkins, MD ${ }^{1}$ Lisa D. Levine, MD ${ }^{1} \quad$ Nathanael C. Koelper, MPH ${ }^{2} \quad$ Celeste Durnwald, MD ${ }^{1}$ \\ ${ }^{1}$ Division of Maternal and Fetal Medicine, Hospital of the University of \\ Pennsylvania, Philadelphia, Pennsylvania \\ 2 Department of Obstetrics and Gynecology, Center for Research on \\ Reproduction and Women's Health, Hospital of the University of \\ Pennsylvania, Philadelphia, Pennsylvania \\ Address for correspondence Maeve Hopkins, MD, Hospital of the \\ University of Pennsylvania, 3400 Spruce Street, Silverstein Building, \\ 2nd Floor, Philadelphia, PA 19104 (e-mail: Hopkins634@gmail.com).
}

Am J Perinatol 2022;39:457-463.

\section{Abstract \\ Keywords \\ - pregnancy \\ - obesity \\ - echocardiogram \\ - hypertension \\ - preeclampsia \\ - maternal morbidity and mortality}

Objective Women with obesity and other comorbidities such as hypertension and diabetes are at an increased risk of preeclampsia and perinatal morbidity. This study evaluates whether screening echocardiogram can identify women with obesity at a higher risk of preeclampsia.

Methods We conducted a retrospective cohort study of women with class III obesity (body mass index [BMI] $\geq 40 \mathrm{~kg} / \mathrm{m}^{2}$ ) and one or more medical comorbidities associated with an increased risk of preeclampsia (such as diabetes, hypertension, and rheumatologic disease) undergoing screening echocardiogram. Abnormal findings were defined as the presence of one or more of the following: diastolic dysfunction, ejection fraction of $\leq 45 \%$, or cardiac chamber enlargement or hypertrophy. Multivariable logistic regression was used to estimate the odds ratio (OR) of gestational hypertension/mild preeclampsia, severe preeclampsia, and any preterm delivery $<37$ weeks associated with abnormal echocardiographic findings when controlling for potential confounders.

Results Of 267 eligible women, 174 (64\%) underwent screening echocardiograms. Sixty-nine women (40\%) had abnormal echocardiograms. Maternal clinical characteristics were similar between women with normal echocardiographic findings and women with abnormal findings. Women with abnormal echocardiograms were more likely to have chronic hypertension ( $78 \mathrm{vs.} 62 \%, p=0.04$ ) and a history of preeclampsia ( 27 vs. $10 \%, p=0.02$ ). After controlling for confounders, women with abnormal echocardiogram were at an increased risk of hypertensive disorders of pregnancy, OR 6.80 (95\% confidence interval $[\mathrm{Cl}] 3.32-13.93, p=0.01)$, and in particular severe preeclampsia, OR 8.77 (95\% Cl 3.90-19.74, $p=0.01$ ).

Conclusion Among pregnant women with class III obesity and medical comorbidities, screening echocardiogram may help identify a subset of women at the highest risk of developing preeclampsia. received

November 5, 2020 accepted after revision October 4, 2021 published online November 23, 2021
DOI https://doi.org/ 10.1055/s-0041-1739520. ISSN 0735-1631.

\footnotetext{
(C) 2021. The Author(s).

This is an open access article published by Thieme under the terms of the Creative Commons Attribution-NonDerivative-NonCommercial-License, permitting copying and reproduction so long as the original work is given appropriate credit. Contents may not be used for commercial purposes, or adapted, remixed, transformed or built upon. (https://creativecommons.org/ licenses/by-nc-nd/4.0/) Thieme Medical Publishers, Inc., 333 Seventh Avenue, 18th Floor, New York, NY 10001, USA
} 


\section{Key Points}

- Women with obesity and comorbid conditions are at a high risk of abnormal echocardiogram.

- Women with obesity, medical comorbid conditions, and abnormal echo are at a high risk of preeclampsia.

- Screening echocardiogram can help identify obese women at the highest risk of severe preeclampsia.

Pregnancy requires complex cardiovascular adaptation. In a normal pregnancy, cardiac output increases by 30 to $50 \%$, primarily through decreased systemic vascular resistance. This leads to reduced mean arterial pressure (MAP), triggering a compensatory increase in the heart rate and stroke volume. ${ }^{1,2}$ Echocardiogram studies in healthy pregnancies have shown an increase in cardiac output, with some correlation with maternal body surface area and fetal birth weight. In a healthy pregnancy, left ventricular (LV) systolic and diastolic function is preserved until term. ${ }^{3}$

Class III obesity, defined by the World Health Organization as a body mass index (BMI) of $40 \mathrm{~kg} / \mathrm{m}^{2}$ or greater, increases the risk of hypertension and cardiac disease outside pregnancy. Class III obesity has been linked to changes in LV morphology including ventricular hypertrophy, as well diastolic dysfunction and enlargement of the left atrium. ${ }^{4}$ On echocardiogram, non-pregnant women with obesity have shown elevated MAP, increased LV mass, but similar cardiac output and diastolic changes compared with non-obese controls. $^{5}$

There are limited data regarding baseline echocardiogram findings in pregnant women with obesity. Studies examining screening echocardiogram in women with obesity show increased LV mass compared with normalweight controls, although they have similar cardiac output and diastolic changes. ${ }^{5-7}$ These studies hypothesized that obese women may not undergo the normal cardiovascular adaptations to pregnancy, however excluding women with coexisting comorbidities such as hypertension and diabetes.

In addition to increasing the risk of cardiac complications outside of pregnancy, class III obesity has also been linked to numerous adverse pregnancy outcomes including hypertensive disorders of pregnancy (HDP). ${ }^{8}$ HDPs, particularly severe preeclampsia, have been associated with echocardiographic abnormalities, including systolic and diastolic dysfunction and left ventricular hypertrophy $(\mathrm{LVH}) .{ }^{9,10}$ Previous data show echocardiogram abnormalities occur at the time of diagnosis of HDP and persist postpartum, ${ }^{11}$ but there are limited data evaluating echocardiogram prior to the diagnosis of HDP, especially among an at-risk population such as hypertensive women with obesity. Our objective is to evaluate the association between abnormal echocardiographic findings and the development of HDP among women with class III obesity. We hypothesize that women with class III obesity and underlying abnormalities on echocardiogram will be at a higher risk of hypertensive diseases of pregnancy compared with women without abnormal echocardiographic findings.

\section{Materials and Methods}

We performed a retrospective cohort study of pregnant women with class III obesity undergoing screening echocardiogram from January 2013 through December 2018 who received prenatal care and delivered within the University of Pennsylvania Hospital System. BMI was calculated from their initial prenatal visit and self-reported height. Class III obesity was defined as BMI $\geq 40 \mathrm{~kg} / \mathrm{m}^{2}$. The study was approved by our institutional review board. Our institution implemented a protocol in 2013 that recommended a screening maternal echocardiogram for class III obese women with one or more medical comorbidity such as preexisting hypertension, diabetes, rheumatologic illness, or prior documented electrocardiogram with LVH.

Individual chart review was performed by a single reviewer (M.H.). Women were excluded if they had multiple gestations (given the additional cardiovascular changes that occur with multiples), underwent echocardiogram due to clinical symptoms, delivered at an outside hospital, or if they had pre-existing cardiac disease (repaired congenital heart disease, known coronary artery disease, or history of myocardial infarction). Our primary outcome of interest was any hypertensive disease of pregnancy. Gestational hypertension and preeclampsia with or without severe features were defined by clinical diagnosis, based on previously published American College of Obstetricians and Gynecologists definitions ${ }^{12}$ and confirmed on chart review. Worsening chronic hypertension (CHTN) was defined as an initiation or uptitration of anti-hypertensive medication during pregnancy in the absence of laboratory blood work abnormalities suggestive of preeclampsia or a medically indicated delivery due to worsening hypertension without an increase in proteinuria. Women diagnosed with worsening CHTN were not diagnosed with a superimposed HDP. Results were stratified by HDP disease severity.

The clinical interpretation of echocardiograms was used for this study with all echocardiograms interpreted by clinical cardiologists at the University of Pennsylvania. An abnormal echocardiogram was defined by previously published expert opinion ${ }^{13,14}$ as LVH (interventricular septum or posterior wall thickness $\geq 11 \mathrm{~mm}$ ), left atrial (LA) enlargement (LA volume index $\geq 35 \mathrm{~cm}^{3} / \mathrm{m}^{2}$ or LA systolic diameter long axis $>3.8 \mathrm{~cm}$ ), right ventricular dilation (size was assessed qualitatively by interpreting cardiologist in standard apical four-chamber view), diastolic dysfunction graded on four stages: normal diastolic function; impaired relaxation with normal or near-normal filling pressures (grade I/IV); impaired relaxation with moderate elevation of filling pressures, pseudonormal filling (grade II/IV); and impaired 
relaxation with marked elevation of filling pressures, restrictive filling (grades III-IV/IV) as previously described, and LV ejection fraction $\leq 45 \%{ }^{15,16}$

Based on the review of the initial 25 subjects, we estimated that the prevalence of abnormal echocardiograms was $30 \%$ of the study population. Based on a fixed sample size of 174 women and an assumed rate of preeclampsia of $20 \%$ in the normal echocardiogram group (based on previously published data in this high-risk group), we would have $80 \%$ power to detect a relative risk of 1.96 with an $\alpha$ of 0.05 .

Categorical variables were compared using Pearson Chisquare or Fisher's exact test, where appropriate. T-test or Mann-Whitney U tests were used to compare continuously measured risk factors, as appropriate. Multivariable logistic regression models were used to control for potential confounders. Variables with a $p<0.2$ from the univariate analysis were considered for inclusion in the final model using backward selection. We tested for effect modification by the known history of CHTN with an interaction term of CHTN and abnormal echocardiogram. Stratified regression models were presented to show the effect modification. A sensitivity analysis was performed limiting the HDP to women with severe preeclampsia. Statistical analysis was performed using Stata 14.2 (College Station, TX).

\section{Results}

- Fig. 1 demonstrates the study population and inclusion. Of 267 women meeting criteria for inclusion, 93 were excluded, 80 of whom (30\%) did not complete the ordered echocardiogram and 13 of whom were excluded due to medical indications as demonstrated in - Fig. 1. In total, 174 women met the study inclusion criteria. Of these, 69 (40\%) had an abnormality on their echocardiogram.

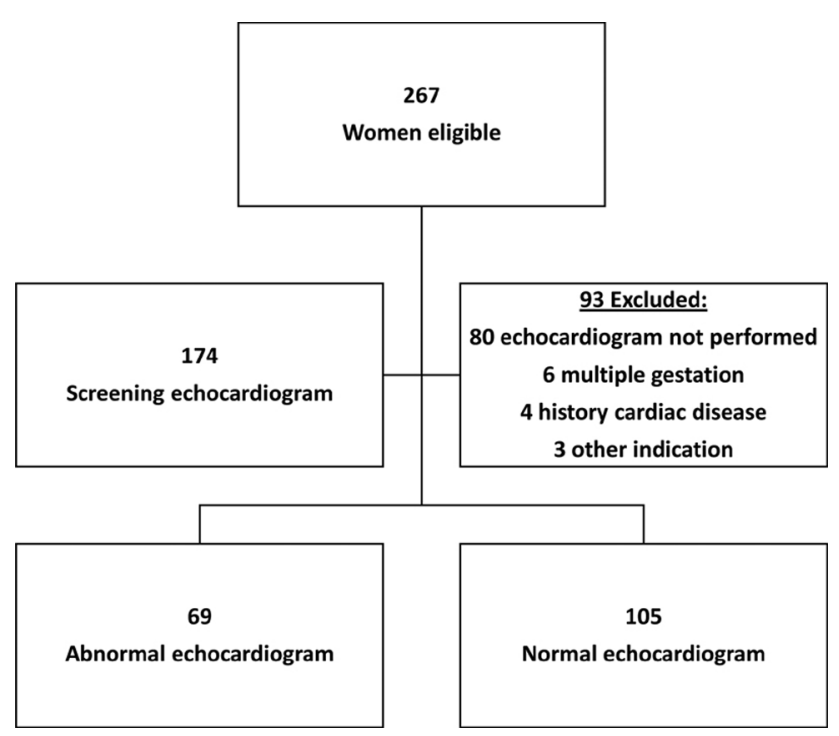

Fig. 1 Flow diagram of study population and echocardiogram completion.
- Table 1 demonstrates the demographic and clinical differences in women with normal versus abnormal echocardiogram. Demographics including age, race, BMI, and preexisting diabetes were similar between the two groups. Of note, over $90 \%$ of our cohorts were self-reported Black race. Women with abnormal echocardiograms were more likely to have CHTN ( $78 \%$ vs. $62 \%, p=0.04$ ), a history of preeclampsia ( $27 \%$ vs. $11 \%, p=0.02$ ), and had the echocardiogram performed at a later gestational age (22.5 vs. 27.1 weeks, $p=0.01$ ).

- Table 2 demonstrates the specific abnormalities found on echocardiogram. The most common abnormality noted on screening echocardiogram was LVH in 48 (70\%) cases. The second most frequent abnormality was left atrial enlargement, followed by diastolic dysfunction. Of the abnormal echocardiograms, 22 women (32\%) had more than one abnormal finding.

The overall rate of a diagnosis of hypertensive disorder of pregnancy in this cohort was $43 \%$ with a significant difference in HDP rates between women with and without an abnormal echocardiogram ( 70 vs. $25 \%, p=0.01$ ), as demonstrated in -Table 3 . This held true when adjusting for confounders including gestational age at echocardiogram and history of CHTN (adjusted odds ratio [aOR] 6.80, 95\% confidence interval [CI] 3.32-13.93), as demonstrated in -Table 4. The interaction term for effect modification by CHTN was significant $(p=0.02)$, and therefore, stratified analyses with the history of CHTN were also performed. When stratifying by CHTN, women with an abnormal echocardiogram have increased odds of developing HDP compared with those without an abnormal echocardiogram (aOR 15.48 95\% CI 5.77-41.53). We did not see a significant association between abnormal echocardiogram and HDP in women without CHTN (-Table 4).

A sensitivity analysis was performed limiting the HDP outcome to women with severe preeclampsia only, shown in -Table 5 . The overall rate of severe preeclampsia for the whole cohort was $32 \%$ with a significant difference between women with and without an abnormal echocardiogram (57 vs. $16 \%, p=0.01$ ). In multivariable analysis, women with abnormal echocardiogram had increased odds of developing severe preeclampsia compared with those without an abnormal echocardiogram, aOR 8.77 (95\% CI 3.90-19.74, $p=0.01$ ). Women with CHTN and abnormal echocardiogram had increased odds for developing superimposed severe preeclampsia (aOR 15.11, 95\% CI 5.33-42.83, $p=0.01$ ) with no difference in women without CHTN. A sensitivity analysis was performed removing women with a history of prior preeclampsia, and the association between abnormal echocardiogram and severe preeclampsia remained (aOR 9.00, 95\% CI 3.67-22.05, $p<0.01$ ). LVH was the most common echocardiogram abnormality, and analysis was performed assessing interventricular septal width as a continuous variable in relation to HDP. Women with severe preeclampsia had overall more interventricular septal thickness compared with women with no HDP (1.15 vs. $1.01 \mathrm{~cm}, p=0.01$ ) as demonstrated in the box plot in -Fig. 2. 
Table 1 Demographic data of study cohort.

\begin{tabular}{|c|c|c|c|c|}
\hline & $\begin{array}{l}\text { Cohort } \\
(N=174)\end{array}$ & $\begin{array}{l}\text { Normal echocardiogram } \\
(N=105)\end{array}$ & $\begin{array}{l}\text { Abnormal echocardiogram } \\
(N=69)\end{array}$ & $p$-Value \\
\hline Age, years & $31.4(5.2)$ & $31.1(5.3)$ & $31.8(5.1)$ & 0.38 \\
\hline Race, $N(\%)$ & & & & 0.13 \\
\hline Black & $162(93)$ & $95(90)$ & $67(97)$ & \\
\hline Other & $12(7)$ & $10(10)$ & $2(3)$ & \\
\hline Delivery BMI, kg/m² & $47.0(44.2-52.3)$ & $47.5(44.3-51.1)$ & $46.3(44.0-54.5)$ & 0.88 \\
\hline Delivery BMI, kg/m² & & & & 0.30 \\
\hline$<50$ & $114(66)$ & 72 (69) & $42(61)$ & \\
\hline $50+$ & $60(34)$ & $33(31)$ & $27(39)$ & \\
\hline Chronic HTN, N (\%) & & & & 0.037 \\
\hline No & $53(32)$ & $38(38)$ & $15(22)$ & \\
\hline Yes & $115(68)$ & $63(62)$ & $52(78)$ & \\
\hline History of PEC, $N(\%)$ & & & & 0.004 \\
\hline No & $89(76)$ & $59(86)$ & $30(63)$ & \\
\hline Yes & $28(24)$ & $10(14)$ & $18(38)$ & \\
\hline Nulliparous & $45(26)$ & $27(26)$ & $18(26)$ & 0.956 \\
\hline Preexisting DM, N (\%) & & & & 0.23 \\
\hline No & $134(80)$ & $79(76)$ & $55(86)$ & \\
\hline Class B & $31(18)$ & $22(21)$ & $9(14)$ & \\
\hline Class $C$ or higher & $3(2)$ & $3(3)$ & $0(0)$ & \\
\hline GA at echocardiogram, weeks & $24.1(17.0-30.1)$ & $22.5(15.1-28.4)$ & $27.1(21.0-31.6)$ & 0.003 \\
\hline
\end{tabular}

Abbreviations: BMI, body mass index; DM, diabetes; GA, gestational age; HTN, hypertension; PEC, preeclampsia.

Note: Gestational age and BMI data expressed as mean \pm SD.

Table 2 Abnormal echocardiogram findings of the study cohort

\begin{tabular}{ll|}
\hline Abnormal echocardiogram finding & $N(\%)$ \\
Left ventricular hypertrophy (LVH) & $48(70 \%)$ \\
Left atrial enlargement & $22(32 \%)$ \\
Diastolic dysfunction & $16(23 \%)$ \\
Right ventricle enlargement & $7(10 \%)$ \\
Reduced ejection fraction (EF) & $3(4 \%)$ \\
Multiple echocardiogram abnormalities & $22(32 \%)$ \\
\hline
\end{tabular}

Note: Percentages do not add to $100 \%$ and represent the percentage of women who had the respective echocardiogram abnormality and some women who had more than one abnormality.

\section{Discussion}

In this study, $40 \%$ of women with class III obesity and comorbid conditions were found to have an abnormal screening echocardiogram. These women had a significantly higher risk of developing a hypertensive disorder of pregnancy compared with those with normal echocardiogram findings. This study demonstrates the potential role of echocardiogram to identify women at an increased risk of worsening hypertension and pregnancy-related cardiac complications. Additionally, the high rate of echocardiogram abnormalities in our cohort suggests that screening echocardiogram can identify a significant number of high-risk women with underlying cardiac abnormalities. This could allow closer cardiac monitoring, cardiology consultation, and targeted postpartum follow-up to intervene in women at a

Table 3 Rates of hypertensive disorders of pregnancy, severe preeclampsia, and preterm delivery

\begin{tabular}{|lllll|}
\hline & Cohort & Normal echocardiogram & \multicolumn{2}{l}{$\begin{array}{l}\text { Abnormal echocardiogram } \\
N=69\end{array}$} \\
& $N=174$ & $N=105$ & $48(70 \%)$ & 0.001 \\
Hypertensive disorder pregnancy (HDP) & $74(43 \%)$ & $26(25 \%)$ & $39(57 \%)$ & 0.001 \\
Severe preeclampsia & $56(32 \%)$ & $17(16 \%)$ & $26(38 \%)$ & 0.006 \\
$\begin{array}{l}\text { Preterm Delivery } \\
(<37 \text { weeks) }\end{array}$ & $46(26 \%)$ & $20(19 \%)$ & & \\
\hline
\end{tabular}




\begin{tabular}{|c|c|c|c|c|c|c|}
\hline \multicolumn{7}{|c|}{ HDP vs. No HDP } \\
\hline & No HDP & HDP & OR (95\% Cl) & $p$-Value & aOR (95\% Cl) & $p$-Value \\
\hline \multicolumn{7}{|l|}{ Echo } \\
\hline Normal & $79(79)$ & $26(35)$ & 1 & & 1 & \\
\hline Abnormal & $21(21)$ & $48(65)$ & $6.95(3.53-13.68)$ & 0.001 & $6.80(3.32-13.93)$ & 0.001 \\
\hline \multicolumn{7}{|l|}{ CHTN } \\
\hline No & $34(35)$ & $19(27)$ & 1 & & 1 & \\
\hline Yes & $63(65)$ & $52(73)$ & $1.48(0.76-2.89)$ & 0.254 & $1.06(0.50-2.25)$ & 0.874 \\
\hline GA at Echo & $23.3(16.9-31.3)$ & $25.3(17-30)$ & $1.01(0.98-1.05)$ & 0.442 & $0.99(0.95-1.03)$ & 0.59 \\
\hline \multicolumn{7}{|c|}{ Severe PEC vs. No HDP } \\
\hline & No HDP & Severe PEC & OR $(95 \% \mathrm{Cl})$ & $p$-Value & aOR $(95 \% \mathrm{Cl})$ & $p$-Value \\
\hline \multicolumn{7}{|l|}{ Echo } \\
\hline Normal & $79(79)$ & $17(30)$ & 1 & & 1 & \\
\hline Abnormal & $21(21)$ & $39(70)$ & 8.63 (4.09-18.19) & 0.001 & 8.77 (3.90-19.74) & 0.001 \\
\hline \multicolumn{7}{|l|}{ CHTN } \\
\hline No & $34(35)$ & $11(20)$ & 1 & & 1 & \\
\hline Yes & $63(65)$ & $43(80)$ & $2.11(0.96-4.61)$ & 0.062 & $1.76(0.73-4.23)$ & 0.206 \\
\hline GA at Echo & $23.3(16.9-31.3)$ & $25(17.1-29.5)$ & $1.01(0.97-1.05)$ & 0.582 & $0.97(0.92-1.12)$ & 0.202 \\
\hline
\end{tabular}

Abbreviations: aOR, adjusted odds ratio; $\mathrm{Cl}$, confidence interval; $\mathrm{OR}$, odds ratio.

Table 5 Odds of hypertensive disorders of pregnancy (HDP) and severe preeclampsia (PEC) stratified by chronic hypertension (CHTN) and gestational age (GA) at echocardiogram

\begin{tabular}{|c|c|c|c|c|}
\hline \multicolumn{5}{|c|}{ HDP vs. No HDP } \\
\hline & \multicolumn{2}{|l|}{ No CHTN } & \multicolumn{2}{|l|}{ CHTN } \\
\hline & aOR (95\% Cl) & $p$-Value & aOR $(95 \% \mathrm{Cl})$ & $p$-Value \\
\hline \multicolumn{5}{|l|}{ Echo } \\
\hline Normal & 1 & & 1 & \\
\hline Abnormal & $1.65(-0.47-5.74)$ & 0.434 & $15.48(5.77-41.53)$ & 0.001 \\
\hline \multicolumn{5}{|l|}{ CHTN } \\
\hline No & - & & - & \\
\hline Yes & - & & - & \\
\hline GA at echo & $1.05(0.97-1.12)$ & 0.222 & $0.95(0.90-1.00)$ & 0.083 \\
\hline \multicolumn{5}{|c|}{ Severe PEC vs. No HDP } \\
\hline & No CHTN & & CHTN & \\
\hline & aOR (95\% Cl) & $p$-Value & aOR $(95 \% \mathrm{Cl})$ & $p$-Value \\
\hline \multicolumn{5}{|l|}{ Echo } \\
\hline Normal & 1 & & 1 & \\
\hline Abnormal & $2.60(0.61-11.13)$ & 0.199 & $15.11(5.33-42.83)$ & 0.001 \\
\hline \multicolumn{5}{|l|}{ CHTN } \\
\hline No & - & & - & \\
\hline Yes & - & & - & \\
\hline GA at echo & $1.00(0.92-1.09)$ & 0.983 & $0.95(0.89-1.01)$ & 0.109 \\
\hline
\end{tabular}

Abbreviations: aOR, adjusted odds ratio; $\mathrm{Cl}$, confidence interval. 


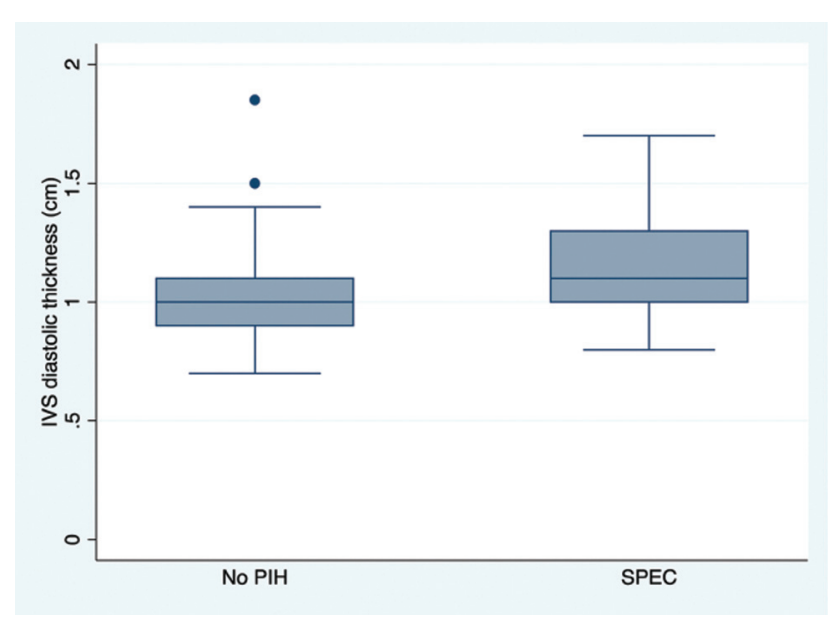

Fig. 2 Boxplot demonstrating interventricular septal (IVS) thickness of severe preeclampsia versus no pregnancy-induced hypertension (PIH). SPEC, severe preeclampsia.

risk of postpartum cardiac morbidity, as well as identify patients who need long-term cardiology care.

Prior studies indicate that LV posterior wall thickness and interventricular septal thickness and mass may be increased in women with obesity. ${ }^{5,6}$ Our rate of LVH of $28 \%$ supports these data, although our data represent a group with a high percentage of underlying CHTN, which puts them at an additional risk of LVH. One study of 40 pregnant women with obesity indicated higher rates of diastolic dysfunction in obese pregnant women compared with normal-weight controls. $^{7}$ Our overall rate of diastolic dysfunction of $9 \%$ was lower than the $40 \%$ identified in this study, indicating that further investigation of diastolic adaptation to the volume load of pregnancy in women with obesity and hypertension is warranted. These data indicate that screening echocardiogram in high-risk pregnant patients with obesity identifies women with underlying cardiac dysfunction and a higher risk of HDP, particularly severe preeclampsia.

Strengths and limitations of this study are as follows. Women received care at a single hospital system that uses a protocol-based approach to both care for obese women and diagnose hypertensive disorders. The study population was primarily Black (93\%) with a relatively high proportion of patients with BMI $\geq 50 \mathrm{~kg} / \mathrm{m}^{2}$ (34\%), which may limit applicability to all clinical settings. However, the importance of evaluating adverse outcomes in this high-risk population cannot be overstated given the extremely high risk of maternal morbidity in this group. Another limitation is the relatively high number of women that did not undergo the recommended screening echocardiogram (30\% of those eligible). The need for insurance prior authorization may be a barrier to obtain an echocardiogram or obtain one on time. This limits sample size and may represent the highest-risk subset of women (if barriers to care contribute to the lack of screening). However, their exclusion would likely bias toward the null hypothesis. Furthermore, our data are not able to elucidate whether it is the echocardiographic abnormalities themselves or the underlying medical issues (particularly CHTN) that ultimately lead to the increased risk of HDP, particularly if the echocardiogram was performed later in pregnancy. Regardless, the identification of these abnormal findings is important to counseling, risk prediction and stratification, labor and delivery management, and postpartum follow-up.

In a high-risk, class III obese population, screening echocardiogram may identify both women with underlying cardiac dysfunction and women at the highest risk of HDP. Abnormal echocardiogram findings may identify women with more severe CHTN or predilection to cardiac morbidity. Given the association with chronic HTN, echocardiogram abnormalities may represent a continuum of pathology. Those with baseline CHTN may be closer to the continuum for diagnostic pathology or abnormalities that are subsequently unmasked with superimposed preeclampsia. Identifying women with abnormal echocardiograms provides an opportunity for earlier intervention to reduce the risk of maternal morbidity and even mortality, by identifying women who need more intensive cardiovascular monitoring intra- and postpartum and women who need a referral to tertiary care centers. At our institution, a collaborative cardiology and obstetrics clinic has been established, and women with abnormal screening echocardiograms are scheduled for postpartum cardiology follow-up after discharge.

Pregnancy adaptation can provide a window to cardiovascular health. Early postpartum follow-up and medical or lifestyle intervention may optimize future pregnancies and contribute to improved lifelong health. Future research should investigate the role of preconception echocardiogram and serial echocardiogram during pregnancy and focus on intervention in postpartum cardiology follow-up as well as medical optimization and appropriate inter-pregnancy interval to optimize outcomes for this at-risk population.

Note

This research was presented as a poster at the American College of Obstetrics and Gynecology (ACOG) meeting in Nashville, TN 2019.

\section{Conflict of Interest}

None declared.

\section{References}

1 Meah VL, Cockcroft JR, Backx K, Shave R, Stöhr EJ. Cardiac output and related haemodynamics during pregnancy: a series of metaanalyses. Heart 2016;102(07):518-526

2 Sanghavi M, Rutherford JD. Cardiovascular physiology of pregnancy. Circulation 2014;130(12):1003-1008

3 Desai DK, Moodley J, Naidoo DP. Echocardiographic assessment of cardiovascular hemodynamics in normal pregnancy. Obstet Gynecol 2004;104(01):20-29

4 Wierzbowska-Drabik K, Chrzanowski L, Kapusta A, et al. Severe obesity impairs systolic and diastolic heart function - the significance of pulsed tissue Doppler, strain, and strain rate parameters. Echocardiography 2013;30(08):904-911

5 Dennis AT, Castro JM, Ong M, Carr C. Haemodynamics in obese pregnant women. Int J Obstet Anesth 2012;21(02):129-134

6 Veille JC, Hanson R. Obesity, pregnancy, and left ventricular functioning during the third trimester. Am J Obstet Gynecol 1994;171(04):980-983 
7 Buddeberg BS, Sharma R, O’Driscoll JM, Kaelin Agten A, Khalil A, Thilaganathan B. Cardiac maladaptation in obese pregnant women at term. Ultrasound Obstet Gynecol 2019;54(03):344-349

8 American College of Obstetricians and Gynecologists. ACOG Committee Opinion, no. 549: obesity in pregnancy. Obstet Gynecol 2012;121:213-217

9 Melchiorre K, Sharma R, Thilaganathan B. Cardiovascular implications in preeclampsia: an overview. Circulation 2014;130(08):703-714

10 Levine LD, Lewey J, Koelper N, et al. Persistent cardiac dysfunction on echocardiography in African American women with severe preeclampsia. Pregnancy Hypertens 2019;17:127-132

11 Melchiorre K, Sutherland GR, Liberati M, Thilaganathan B. Preeclampsia is associated with persistent postpartum cardiovascular impairment. Hypertension 2011;58(04):709-715

12 American College of Obstetricians and Gynecologists. ACOG Practice Bulletin no. 202: gestational hypertension and preeclampsia. Obstet Gynecol 2019;133(01):e1-e25
13 Lang RM, Badano LP, Mor-Avi V, et al. Recommendations for cardiac chamber quantification by echocardiography in adults: an update from the American Society of Echocardiography and the European Association of Cardiovascular Imaging. Eur Heart J Cardiovasc Imaging 2015;16(03):233-270

14 Redfield MM, Jacobsen SJ, Burnett JC Jr, Mahoney DW, Bailey KR, Rodeheffer RJ. Burden of systolic and diastolic ventricular dysfunction in the community: appreciating the scope of the heart failure epidemic. JAMA 2003;289(02):194-202

15 Lanier GM, Vaishnava P, Kosmas CE, Wagman G, Hiensch R, Vittorio TJ. An update on diastolic dysfunction. Cardiol Rev 2012;20(05):230-236

16 Nagueh SF, Smiseth OA, Appleton CP, et al. Recommendations for the evaluation of left ventricular diastolic dysfunction by echocardiography: an update from the American Society of Echocardiography and the European Association of Cardiovascular Imaging. J Am Soc Echocardiogr 2016;29(04):277-314 